\title{
Kernos
}

Revue internationale et pluridisciplinaire de religion grecque antique

19 | 2006

Varia

\section{Cornelia ISLER-KERÉNYI, Civilizing Violence. Satyrs on 6th-Century Greek Vases}

\section{Véronique Dasen}

\section{OpenEdition \\ Journals}

\section{Édition électronique}

URL : https://journals.openedition.org/kernos/483

DOI : $10.4000 /$ kernos.483

ISSN : 2034-7871

\section{Éditeur}

Centre international d'étude de la religion grecque antique

\section{Édition imprimée}

Date de publication : 1 janvier 2006

Pagination : 475-477

ISSN : 0776-3824

\section{Référence électronique}

Véronique Dasen, «Cornelia IsLeR-KERÉNYı, Civilizing Violence. Satyrs on 6th-Century Greek Vases », Kernos [En ligne], 19 | 2006, mis en ligne le 24 mai 2011, consulté le 24 août 2022. URL : http:// journals.openedition.org/kernos/483; DOI : https://doi.org/10.4000/kernos.483 
In all, this is a very useful book to have at hand when working with Greek sacrifices, since it includes such a large amount of evidence, both thoroughly described and illustrated. One will find almost anything one looks for, either in the catalogues or in the footnotes, and certain items included are previously unpublished. In this sense, it definitely constitutes an addition to van Straten's Hierà kalá. Still, for the actual interpretation and discussion of the evidence, there is a curious lack of interest in the heart of the matter, Greek religion. Gebauer's focus on and interest in the iconography are his prime concern. His urge to demonstrate the validity of his method, to identify the specific types of figures and scenes used by the vase-painters, is so strong that one almost gets the impression he could have chosen to apply it to any topic. This is a descriptive study of Greek animal sacrifices on Attic vases which is not very interested in analyzing Greek cult. To give one specific example, there is no real discussion to what extent rituals such as theoxenia and trapezomata, often used to modify animal sacrifices of the thysia kind, may be distinguished in the pictorial evidence, either as direct representations or as more discrete references. The term trapezomata is not even found in the index. An interesting group of scenes showing women seated at tables with meat, which may relate to these rituals (p. 337, n. 1331, and p. 486, n. 1844), is briefly mentioned, but it would certainly have been interesting to have the meaning of this motif developed within the wider iconographic context. After going through such a large amount of material, Gebauer would have been in an excellent position to approach such intricate issues. Moreover, Gebauer takes the Olympian/chthonian model as valid for the basic theoretical approach to the evidence. A greater awareness of the difficulties inherent in the concepts Olympian and chthonian would have improved and deepened the quality of the analysis. As it is now, at numerous instances he simply explains an observation by resorting to Olympian/chthonian stereotypes, be it the choice of white and black animals (p. 19), the height of altars (p. 373) or the blood at sphagia sacrifices being poured into a bothros (p. 255). To consider oath sacrifices and purifications as chthonian rituals is certainly to transgress the valid use of this terminology (p. 255). More surprisingly, on p. 476 (and n. 1794) he suddenly declares the distinction between Olympian and chthonian sacrifices to be a modern construct. Finally, there is also a clear preference for using German scholarship within the discussion, leaving out some important studies, though some of these may have appeared too late to be included?

In the end, hold on to your copy of van Straten's Hierà kalá, but do not hesitate to get Gebauer's Pompe und Thysia as well.

Gunnel Ekroth

(University of Stockholm)

Cornelia IsLER-KERÉNYI, Civilizing Violence. Satyrs on 6th-Century Greek Vases, Göttingen/Fribourg, Vandenhoeck \& Ruprecht/Academic Press, 2004. 1 vol. $15,5 \times 23 \mathrm{~cm}, 123$ p. (Orbis Biblicus et Orientalis, 208). ISBN : 3-525-530668/3-7278-1497-7.

Dans la foulée de son étude sur l'iconographie de Dionysos à l'époque archaïque (Dionysos nella Grecia arcaica. Il contributo delle immagini 2001), C. Isler-Kerényi

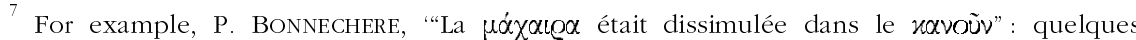
interrogations', REG 101 (1999), p. 21-35, and M. HALM-TISSERANT, "Le paysage sacré dans la peinture de vases grecque", Ktema 24 (1999), p. 243-250, as well as the works referred to above in n. 3 and 4 .
} 
nous offre la synthèse de ses réflexions sur l'origine, la nature et la fonction des satyres dans la céramique grecque du $v^{e}{ }^{e} s$. et du début du v ${ }^{e} s$. L'ambivalence marque ces figures hybrides, mêlant corps d'humain et d'équidé, marginalisées par un comportement sexuel débridé, tout en se situant avec Dionysos au cœur de l'expérience grecque du vin, du symposion, et par là de la société elle-même. Comment comprendre le contraste entre la profusion des images et le silence des textes? Quel rapport entretiennent les satyres des arts figurés et ceux du drame satyrique? Posées en introduction, ces questions sous-tendent l'ouvrage centré sur une iconographie qui donne à voir un univers démonique où la turbulence du satyre décline différentes manières d'intégrer le désordre (plutôt que la violence) de façon constructive dans la polis. Les deux premiers chapitres explorent l'émergence de la figure du satyre au début $\mathrm{du} \mathrm{VI}^{\mathrm{e}} \mathrm{s}$. Les plus anciennes représentations mettent en scène le tempérament contrasté du personnage, tantôt créature sauvage à l'affût de nymphes, tantôt compagnon au service de Dionysos, escortant le retour d'Héphaïstos.

Les trois chapitres suivants analysent différents types de scènes où les satyres transposent sur le plan surnaturel l'expérience religieuse des humains : cômos mêlant satyres et fêtards dont le corps s'apprête à se transformer au contact de Dionysos (chapitre 3), thiase où le satyre salue l'épiphanie du dieu et de sa compagne (chapitre 4), ambiguïtés du cortège du "retour d'Héphaïstos » où l'identité des acteurs, entre mythe et rite, se trouble (chapitre 5). Dans tous ces exemples, le satyre ne cesse d'interpeller le spectateur. Il joue le rôle de médiateur, abolissant la distance entre le divin et l'humain, réplique démonique de l'homme métamorphosé sous l'emprise du dieu. Sous le règne de Pisistrate, vers 540, apparaît le motif de la vendange divine (chapitre 6) où des satyres, parfois miniaturisés, récoltent le raisin et s'activent à le transformer en vin, incarnant une technè collective qui opère le passage de la sauvagerie à la civilisation. La grappe que tient le dieu renvoie métaphoriquement à sa mise à mort, puis à sa renaissance sous la forme du vin offert aux humains. Le dernier chapitre (7) est consacré à l'évolution du personnage dans la figure rouge, quand le poète Pratinas «invente » le genre du drame satyrique. Sur la céramique peinte, la perméabilité du monde des satyres avec celui des humains devient de plus en plus manifeste. Les satyres se mêlent peu à peu de manière étendue aux activités des citoyens, se substituant aux humains dans les rues de la cité, au gymnase, à la guerre, comme les satyres de l'espace théâtral, que l'on trouve intégrés à des situations non dionysiaques. Ce rôle essentiel de miroir du monde des mortels explique sans doute l'absence des satyres dans les récits mythiques en dépit de leur extraordinaire popularité dans les arts figurés.

Bien que la figure du satyre se prête dans la cité à toutes sortes de jeux visuels qui auraient mérité un développement, l'A. n'évoque pas cette dimension ludique. Plus sensible au souci de replacer l'imagerie dans son contexte historique, C.I.-K. propose plusieurs liens intéressants entre les développements de l'iconographie et les événements de l'époque archaïque. La série de scènes dépeignant le retour d'Héphaïstos sur l'Olympe est ainsi mise en rapport avec les réformes de Solon. L'imagerie semble transposer sur le plan mythique la volonté d'intégrer à la cité les exclus du système aristocratique, tels les exilés, et de promouvoir la classe des artisans (ch. 2 et 5). De même, la création et le développement du motif des vendanges pourraient faire écho à la politique de valorisation du monde rural, de Pisistrate aux réformes de Clisthènes. En résumé, C.I.-K. nous offre une synthèse utile sur un sujet familier mais rarement abordé pour lui-même. Illustré de dessins, l'ouvrage est complété par une bibliographie succincte et des indices. - Une absence est à relever, dans la mesure où l'ouvrage paraît dans la série Orbis Biblicus Orientalis, consacrée à l'iconographie proche- 
orientale. Dans son exposé sur les origines de la figure du satyre dans l'art grec archaïque, l'A. n'a pas relevé l'existence d'une série d'études qui lui auraient apporté une clé d'interprétation. Il s'agit de travaux sur l'influence de la figure du dieu nain Bès, attesté en Égypte dès le Moyen Empire, puis largement diffusé dans les cultures environnantes (Palestine, Phénicie, Chypre), qui présente de nombreuses analogies avec la figure émergente du satyre à l'époque orientalisante: hybridité, pilosité, calvitie, faciès au nez retroussé, sans oublier la multiplicité, car le nom Bès s'applique de manière générique à une troupe de figures démoniques d'apparence identique, comme les Silenoi du Vase François. W. Helck («Phönizische Dämonen im frühen Griechenland », $A A, 1987$, p. 445-447) et P. Blome («Phönizische Dämonen auf einem attischen Krater, $A A, 1985$, p. 573-579) ont ainsi démontré l'emprunt d'un prototype phénicien, dérivé de l'iconographie de Bès, pour les "proto-silènes » d'un cratère proto-attique (fig. 1). Le point sur la transmission du motif de la calvitie est fait dans la thèse de $\mathrm{P}$. Birchler Emery sur «L'iconographie de la vieillesse à l'époque archaïque » (Université de Genève, 2004, sous presse). Les champs de compétence et les modes d'action des Bès et des satyres présentent d'autres similarités: musique, danse, protection de la sexualité, des femmes et des jeunes enfants. Les petites figurines en terre cuite de « démons ventrus » sont un bon exemple d'une autre forme d'adaptation grecque de l'image protectrice de Bès, mais limitée à l'époque archaïque (ca 575-520) (U. SinN, "Zur Wirkung des ägyptischen 'Bes' auf die griechische Volksreligion », in D. Metzler et al. (éds), Antidoron, Festschrift für J. Thimme zum 65. Geburtstag am 26. Sept. 1982, Karlsruhe, 1983, p. 87-94). Les statuettes de Silène bientôt les remplacent dans les tombes d'enfant. Les dieux nains égyptiens incarnent aussi un principe de croissance et de régénération, comme le sont à leur manière les petits satyres vendangeurs des peintres de figure noire (ch. 6, p. 75). Par contamination, l'imagerie égyptienne de Basse-Epoque va progressivement doter les Bès des grands phallus des satyres grecs $(c f$. la présence à Naucratis de vases à figures noires avec des satyres; $c h$ 1, figs 6 et 7); l'influence grecque sur l'image de Bès a été relevée sur les intailles gréco-puniques par A.M. BISI, «Da Bes a Herakles, RStFen 8 (1980), p. 19-42 (de manière plus générale, sur l'iconographie des Bès et Ptah-Patèques et ses rapports avec l'imagerie grecque, on me permettra de citer V. DASEN, Dwarfs in ancient Egypt and Greece, Oxford, 1993, spéc. 77-83 sur la Basse-Époque égyptienne, 200-204 sur les démons kourotrophes, qui font également référence aux Ptah-Patèques).

Véronique Dasen (Université de Fribourg)

María Cruz CARdete Del Olmo, Paisajes mentales y religiosos. La frontera suroeste arcadia en épocas arcaica y clásica, Oxford, 2005. 1 vol. $21 \times 29,5$ cm, 242 p. (BAR International Series, 1365). ISBN : 1-84171-701-0.

L'ouvrage de C. s'inscrit dans la lignée de recherches actuelles qui caractérisent le paysage non seulement par ses traits de géographie physique, mais comme «un système de significations qui intègre des facteurs économiques, sociaux, politiques et symboliques »; le paysage, selon l'A., c'est la manière dont une société et un peuple «vivent et comprennent le monde », d'où le titre de l'ouvrage : "Paysages mentaux et religieux ». À part ce thème de réflexion, une autre idée sous-tend le travail : retrouver le passé sans le reconstruire différemment de ce qu'il était dans l'antiquité est une gageure; l'A. veut essayer de relever le défi à partir de l'étude d'une région limitée, la «frontière » sud-ouest de l'Arcadie à l'époque archaïque et classique. Par frontière, elle 